Adriano Tachibana

\title{
OTIMIZAÇÃO DE PROTOCOLO DE AQUISIÇÃO DE ALTA VELOCIDADE PARA ANGIOTOMOGRAFIA DA AORTA ABDOMINAL, ARTÉRIAS ILÍACAS E ARTÉRIAS DOS MEMBROS INFERIORES UTILIZANDO EQUIPAMENTO COM 160 FILEIRAS DE DETECTORES
}

Tese apresentada à Sociedade Beneficente Israelita Brasileira Albert Einstein para obtenção do título de Doutor em Ciências da Saúde. 
Adriano Tachibana

\title{
OTIMIZAÇÃO DE PROTOCOLO DE AQUISIÇÃO DE ALTA VELOCIDADE PARA ANGIOTOMOGRAFIA DA AORTA ABDOMINAL, ARTÉRIAS ILÍACAS E ARTÉRIAS DOS MEMBROS INFERIORES UTILIZANDO EQUIPAMENTO COM 160 FILEIRAS DE DETECTORES
}

\begin{abstract}
Tese apresentada à Sociedade Beneficente Israelita Brasileira Albert Einstein para obtenção do título de Doutor em Ciências da Saúde.
\end{abstract}

Orientador: Prof. Dr. Nelson Wolosker 
Tachibana, Adriano

Otimização de protocolo de aquisição de alta velocidade para angiotomografia da aorta abdominal, artérias ilíacas e artérias dos membros inferiores utilizando equipamento com 160 fileiras de detectores / Adriano Tachibana. -- São Paulo, 2017. $x i, 28$ f., il.

Tese (Doutorado) - Sociedade Beneficente Israelita Brasileira Albert Einstein. Instituto Israelita de Ensino e Pesquisa Albert Einstein. Programa de Pós-Graduação em Ciências da Saúde.

Título em inglês: Optimized protocol for fast CT angiography of the aorta, iliac and lower limbs arteries using 160-row detector equipment.

1. Angiografia por tomografia computadorizada. 2. Extremidade inferior. 3. Doença arterial periférica. 4. Técnicas de diagnóstico cardiovascular. 5. Meios de contraste.

NLM - WN 206

Elaborada pelo Sistema Einstein Integrado de Bibliotecas 
SOCIEDADE BENEFICENTE ISRAELITA BRASILEIRA ALBERT EINSTEIN

Coordenador do Curso de Pós-Graduação: Prof. Dr. Luiz Vicente Rizzo 
Adriano Tachibana

\section{OTIMIZAÇÃO DE PROTOCOLO DE AQUISIÇÃO DE ALTA VELOCIDADE PARA ANGIOTOMOGRAFIA DA AORTA ABDOMINAL, ARTÉRIAS ILÍACAS E ARTÉRIAS DOS MEMBROS INFERIORES UTILIZANDO EQUIPAMENTO COM 160 FILEIRAS DE DETECTORES}

Presidente da banca: Prof. Dr. Nelson Wolosker

BANCA EXAMINADORA

Membros titulares

Dr. Fábio Sândoli Brito Jr.

Prof. Dr. Antonio Eduardo Zerati

Prof. Dr. Miguel José Francisco Neto

Membros suplentes

Prof. Dr. Marcel Liberman

Dr. Carlos Augusto Ventura Pinto

Aprovada em: 30/10/2017. 


\section{Dedicatória}

Aos meus pais, Arnaldo e Lidia, minhas filhas Mariana e Mei, e à Bruna, minha esposa e eterna companheira. 


\section{Agradecimentos}

Aos meus pais, Arnaldo e Lidia, que nunca mediram esforços para dar o melhor aos filhos e por terem a visão do valor da educação.

Às minhas filhas, Mariana e Mei, alegria da minha vida e exemplo de força e superação.

À Bruna, minha esposa e meu grande amor, companheira para vida, incansável na tarefa de me fazer melhor a cada dia.

Ao Dr. Marcelo Funari que, com sabedoria, inspira e desenvolve o lado bom das pessoas com quem convive.

Ao Dr. Miguel José Francisco Neto, que é um exemplo de bondade, amizade e trabalho.

Ao Dr. Marcos Queiroz, amigo, conselheiro e grande motivador.

Aos médicos do Departamento de Diagnósticos por Imagem que, com extrema competência e profissionalismo, fazem da nossa especialidade uma arte.

Aos biomédicos da Tomografia que formam um grupo de alto desempenho, unidos por amizade e que puderam fazer desta tese realidade.

Ao Dr. Nelson Wolosker, admirado orientador, que soube compreender, conduzir e intervir com precisão cirúrgica durante a realização deste trabalho. 


\section{Sumário}

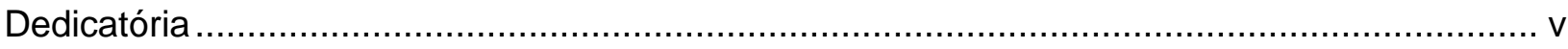

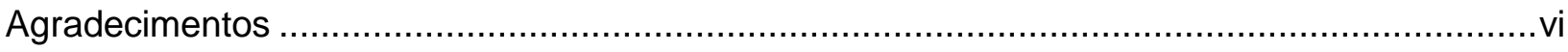

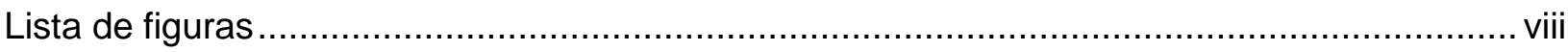

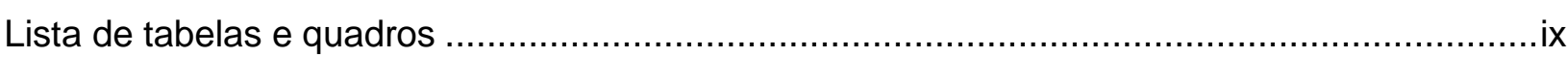

Lista de abreviaturas e símbolos ...............................................................................

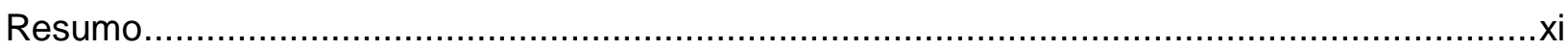

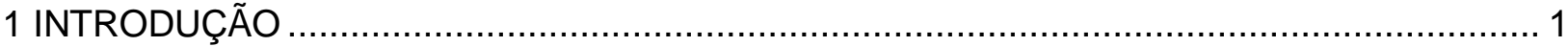

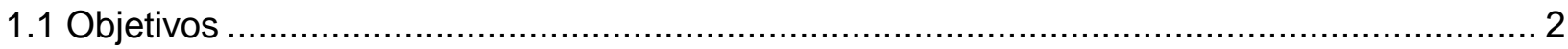

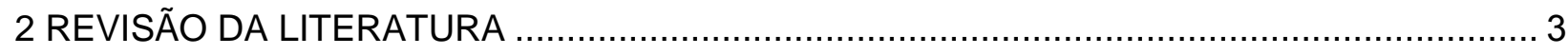

2.1 Evolução tecnológica da tomografia computadorizada e sua relação com o exame de

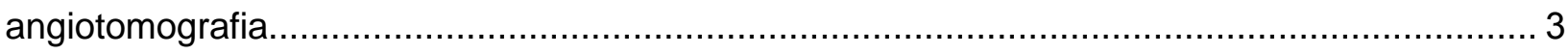

2.2 Fisiologia e farmacocinética do contraste iodado e sua aplicação em angiotomografia ........ 6

2.3 A construção de protocolos otimizados para angiografia por tomografia computadorizada da aorta abdominal, artérias ilíacas e artérias dos membros inferiores com a utilização dos conhecimentos técnicos da fisiologia e farmacocinética do contraste e ajustes técnicos do

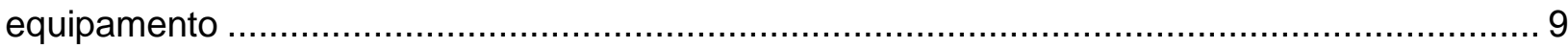

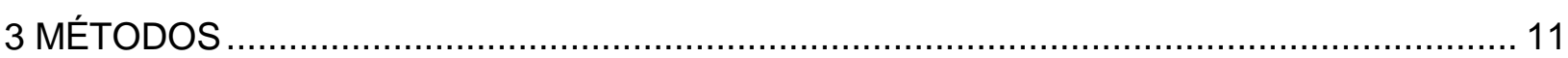

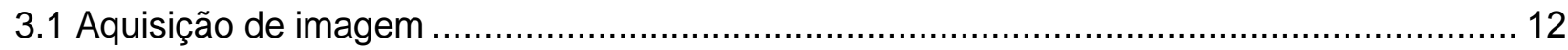

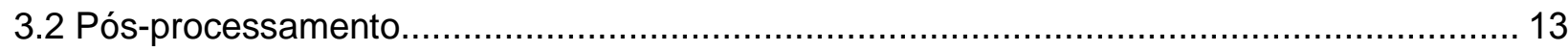

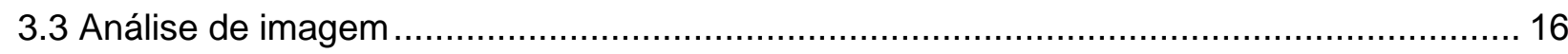

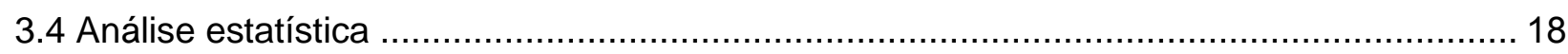

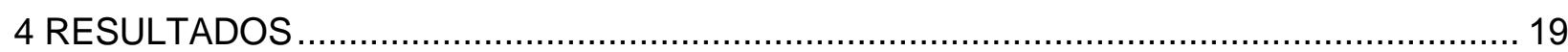

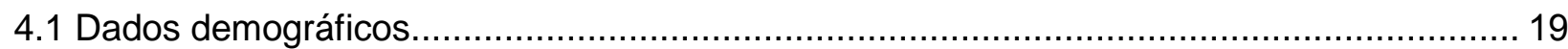

4.2 Volume de contraste e tempo de aquisição das imagens ................................................ 19

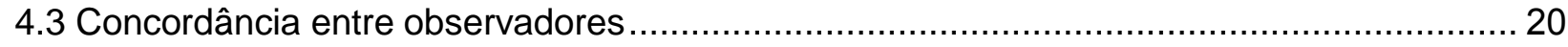

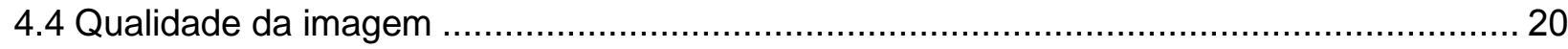

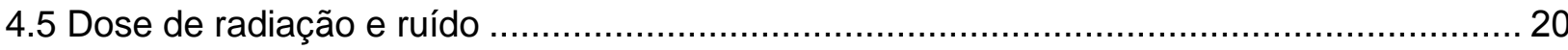

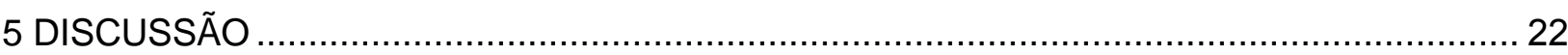

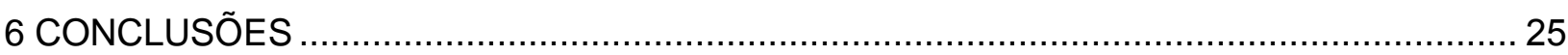

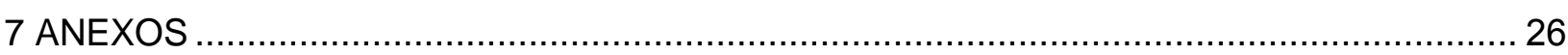

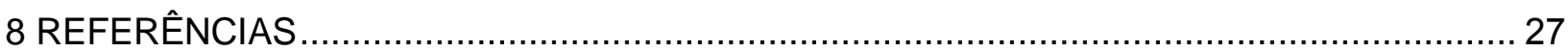

Abstract 


\section{Lista de figuras}

Figura 1. Representação esquemática das gerações de equipamentos de tomografia .4 Figura 2. Reconstrução sagital de angiotomografia realizada em equipamento helicoidal no plano da aorta torácica (seta amarela) e esôfago (seta vermelha).............5 Figura 3. Esquema representando um equipamento de tomografia computadorizada de

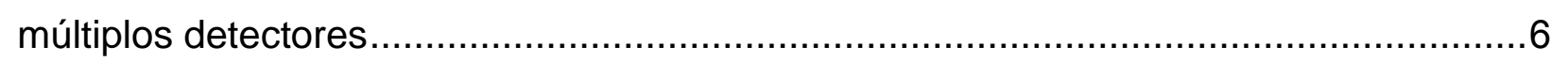

Figura 4. Variação da atenuação vascular $(\Delta \mathrm{UH})$ durante aquisição angiotomográfica arterial em dois tipos de injeção ................................................................... Figura 5. Variação da atenuação vascular $(\Delta U H)$ e o efeito da injeção bifásica (com dois fluxos diferentes de injeção) ......................................................................... Figura 6. Reconstruções tridimensionais em renderização volumétrica de angiografia por tomografia de paciente estudado com protocolo padrão $(A)$ e outro paciente avaliado com protocolo de alta velocidade de aquisição $(B)$......................................15 Figura 7. Reconstruções em projeção de intensidade máxima de angiografia por tomografia dos mesmos pacientes da figura 6 , estudados com protocolo padrão (A) e outro avaliado com protocolo de alta velocidade de aquisição $(\mathrm{B})$.............................16 


\section{Lista de tabelas e quadros}

Tabela 1. Parâmetros do protocolo para aquisição de alta velocidade com 160 fileiras de detectores (Grupo 1) e padrão com 64 fileiras de detectores (Grupo 2) 12

Tabela 2. Protocolo de injeção trifásica para aquisição de alta velocidade com 160

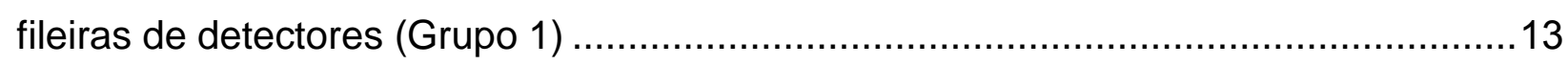

Tabela 3. Protocolo de injeção bifásica para aquisição padrão de 64 fileiras de

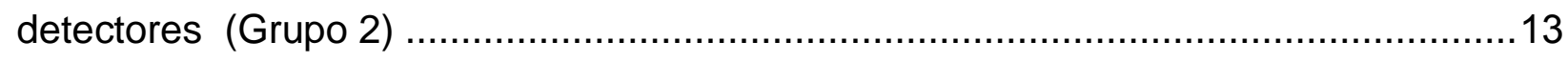

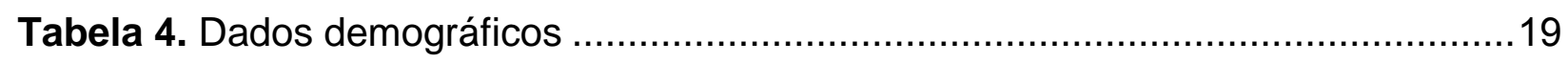

Tabela 5. Resumo dos resultados (análises por paciente) ......................................21

Tabela 6. Resumo dos resultados (análises por segmentos vasculares) ....................21

Quadro 1. Escala de quatro pontos para análise qualitativa ...................................17 


\section{Lista de abreviaturas e símbolos}

$\begin{array}{ll}\Delta \text { UH } & \text { Variação de unidades Hounsfield } \\ \text { 3D } & \text { Tridimensional } \\ \text { CT } & \text { Tomografia computadorizada (Computed Tomography) } \\ \text { CTA } & \text { Angiografia por tomografia computadorizada (Computed Tomography } \\ & \text { Angiography) } \\ \text { DLP } & \text { Produto Dose Comprimento (Dose Lenght Product) } \\ \text { kg } & \text { Quilograma } \\ \text { mg } & \text { Miligrama } \\ \text { mGy.cm } & \text { MiliGray.centrímetros } \\ \text { MIP } & \text { Projeção de intensidade máxima (Maximum intensity projection) } \\ \text { mL } & \text { Mililitro } \\ \text { PACS } & \text { Sistema de Comunicação e Arquivo de Imagens (Picture Archiving and } \\ & \text { Communication System) } \\ \text { ROI } & \text { Região de interesse (Region of interest) } \\ \text { S } & \text { Segundos } \\ \text { UH } & \text { Unidades Hounsfield } \\ \text { VR } & \text { Renderização de volume (Volume rendering) } \\ \end{array}$




\section{Resumo}

Objetivo: Demonstrar ser possível a aplicação de nova técnica de aquisição de alta velocidade em angiotomografia, utilizando a máxima capacidade de equipamento com 160 fileiras de detectores, combinada com injeção multifásica de contraste e comparála com a técnica padrão para exames de angiotomografia da aorta abdominal, artérias ilíacas e artérias dos membros inferiores em relação à qualidade diagnóstica e realce arterial. Comparar a dose de radiação e o ruído na imagem das duas técnicas (objetivo secundário). Métodos: Foi conduzido um estudo prospectivo randomizado com 30 voluntários para comparar a qualidade da imagem (escala subjetiva de 1 a 4), a contrastação arterial (em unidades Hounsfield), a dose de radiação (em mGy.cm) e o ruído na imagem (em desvios padrão da média da atenuação) em dois grupos. Um grupo composto pelo novo protocolo de aquisição rápida (Grupo 1) e o outro de aquisição amplamente aceita, neste estudo chamado de aquisição lenta (Grupo 2). Resultados: Foram incluídos 15 pacientes no Grupo 1 e estudados 187 segmentos arteriais e outros 15 pacientes no Grupo 2 em que foram estudados 190 segmentos. Não houve diferença entre os grupos em relação à qualidade da imagem (nota média de 3,60 \pm 0,21 para o Grupo 1 e 3,62 $\pm 0,21$ para o Grupo 2). Houve maior qualidade diagnóstica para Grupo 1 (99,5\% comparado a 96,4\%), embora tenha ocorrido menor realce vascular $(426,85 \mathrm{UH}$, comparado a $517,67 \mathrm{UH})$. Houve tendência a maior radiação para o Grupo 1 (média de 1296,99 \pm 377,44mGy.cm comparado a 1082,69 \pm 279,74mGy.cm) e menor ruído (20,66 $\pm 2,94$ comparado a 25,21 $\pm 3,21)$. Conclusão: O protocolo proposto, otimizado para realizar angiotomografia de alta velocidade da aorta abdominal, artérias ilíacas e artérias dos membros inferiores possui maior qualidade diagnóstica que o protocolo padrão. Entretanto, foi observado menor realce vascular sem comprometer a qualidade da imagem e tendência a maior dose de radiação resultando em menor ruído nas imagens. Deve ser utilizado em pacientes selecionados que podem se beneficiar de uma aquisição mais rápida. 


\section{INTRODUÇÃO}

A angiotomografia ou angiografia por tomografia computadorizada (CTA - Computed Tomography Angiography) juntamente com a angiografia por ressonância magnética substituiu a angiografia digital entre radiologistas e cirurgiões vasculares para o diagnóstico da doença arterial periférica. ${ }^{(1-6)}$ A acurácia da CTA depende fortemente de técnica de aquisição apropriada, que inclui a escolha certa do volume de contraste administrado, fluxo de injeção e velocidade de aquisição. ${ }^{(7-9)}$

$O$ desenvolvimento de equipamentos com maior número de fileiras de detectores permitiu a realização de aquisições mais extensas e mais rápidas, reduzindo consideravelmente os artefatos decorrentes do movimento do paciente e a dose total de radiação. Além disso, os avanços nas técnicas de pós-processamento das imagens com reformatações multiplanares e 3D melhoraram consideravelmente as capacidades clínicas da CTA. ${ }^{(10,11)}$

Apesar desses avanços, a varredura da CTA de membros inferiores ainda deve ser de baixa velocidade (não se utilizando do potencial máximo do equipamento), devido à progressão relativamente lenta do contraste através do sistema arterial. A combinação de aquisições mais rápidas com os métodos atuais de injeção de contraste resulta em contrastação não homogênea das artérias dos membros inferiores, obtendo-se imagens não diagnósticas. No entanto, propomos que um tempo de injeção de contraste mais longo que o habitual (cerca de 5 a $10 \%$ maior), aliado a injeção multifásica possa fornecer um bolo de contraste mais homogêneo e de maior duração que, juntamente com aquisição tomográfica mais tardia e mais rápida, possa resolver o problema da não homogeneidade nas imagens obtidas, relacionada à variabilidade entre pacientes. ${ }^{(12)}$

Atualmente, não há estudos comparando a qualidade diagnóstica dos exames realizados usando os protocolos de CTA amplamente aceitos, com tempos de aquisição mais lentos ${ }^{(8)}$ e os protocolos de aquisição mais rápidos em combinação com a administração de contraste aprimorada, que utilizem a capacidade total dos equipamentos mais avançados. 


\subsection{Objetivos}

1. Demonstrar ser possível a aplicação de nova técnica de aquisição de alta velocidade em angiotomografia, utilizando a máxima capacidade de equipamento com 160 fileiras de detectores, combinada à injeção multifásica de contraste;

2. Comparar duas técnicas de aquisição angiotomográfica arterial de membros inferiores, sendo uma padrão e outra de alta velocidade em relação à qualidade diagnóstica e realce arterial;

3. Comparar a dose de radiação e o ruído nas imagens das duas técnicas. 


\section{REVISÃO DA LITERATURA}

\subsection{Evolução tecnológica da tomografia computadorizada e sua relação com o exame de angiotomografia}

A tomografia computadorizada (CT - Computed Tomography) como método de diagnóstico por imagem teve seu início em 1971 e se tornou um dos testes mais solicitados no mundo atualmente. ${ }^{(13,14)}$

O primeiro equipamento (EMI Mark I), construído sob supervisão do seu idealizador sir Godfrey Hounsfield, era capaz de realizar imagens apenas da cabeça através de um estreito feixe de raios- $X$ e um detector que desempenhavam movimentos de rotação-translação. ${ }^{(15)} A$ reconstrução das imagens foi possível graças aos estudos sobre atenuação dos feixes de raios- $X$ desenvolvidos por Allan Cormack. ${ }^{(16)}$ Foram chamados de equipamentos de primeira geração e demoravam 4,5 minutos para completar uma imagem da cabeça. ${ }^{(17)}$

Os tomógrafos de segunda geração aprimoraram o conceito inicial e foram construídos com vários detectores que poderiam adquirir vistas simultâneas a cada movimento de rotação e translação. Esta inovação reduziu significativamente o tempo para aquisição, conseguindo-se obter uma imagem em menos de 20 segundos. ${ }^{(17)}$

A terceira geração introduziu a geometria de rotação-rotação, na qual a fonte de raios- $X$ e os detectores rodavam em conjunto ao redor do paciente. Esta nova geometria, associado a um feixe de raios-X em forma de "leque", tornou possível a realização da mesma imagem dos tomógrafos da primeira geração em menos de 5 segundos. ${ }^{(17)}$

$\mathrm{Na}$ tentativa de se ganhar ainda mais eficiência, a mesma geometria dos equipamentos de terceira geração foi aplicada para a construção da quarta geração, que trazia como novidade detectores estáticos completando toda a volta ao redor do paciente. Neste equipamento, somente a fonte de raios- $X$ desempenhava 0 movimento de rotação. Na prática, esta geração não se mostrou mais eficiente que a anterior, além do menor aproveitamento dos detectores (apenas um quarto deles é utilizado simultaneamente). O projeto da quarta geração foi abandonado, não existindo equipamentos comerciais com esta tecnologia. ${ }^{(17)}$ 
Percebe-se que as inovações trazidas pelas quatro gerações de tomógrafos se empenharam em reduzir o tempo necessário para se obter uma imagem no plano axial. Uma grande limitação da tomografia até este ponto do desenvolvimento é a resolução no eixo z (longitudinal). As imagens axiais não podiam ser agrupadas em um volume tridimensional devido à descontinuidade das mesmas.

A figura 1 demonstra em forma de esquema a evolução dos equipamentos desde a primeira até a quarta geração.
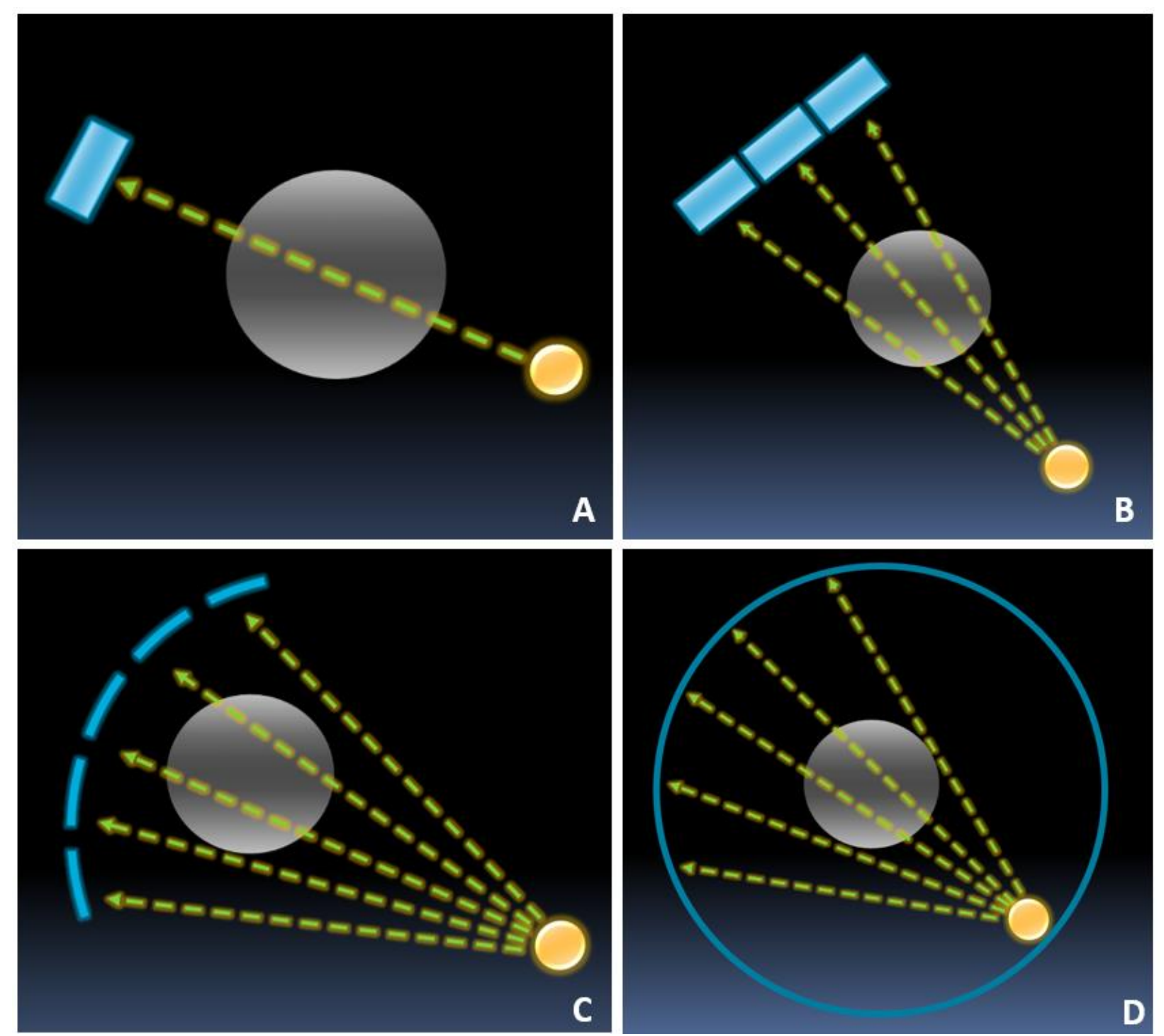

A, B, C e D respectivamente representam tomógrafos de primeira, segunda, terceira e quarta gerações. Fonte: Adaptado de Heijenbrok-Kal MH, Kock MC, Hunink MG. Lower extremity arterial disease: multidetector CT angiography meta-analysis. Radiology. 2007;245(2):433-9. ${ }^{(3)}$

Figura 1. Representação esquemática das gerações de equipamentos de tomografia

Por volta de 1990, o desenvolvimento da tecnologia de aquisição espiral ou helicoidal revolucionou a CT. Foi um aprimoramento da tecnologia dos 
tomógrafos de terceira geração. A técnica consiste em movimentar o paciente através do equipamento, enquanto simultaneamente se realiza o escaneamento contínuo através da rotação do conjunto fonte de raios-X e detector. Os dados obtidos constroem um verdadeiro volume tridimensional que pode ser manipulado por métodos computacionais e apresentado em diversos planos.

Com esta tecnologia, a limitação da resolução longitudinal (eixo z) foi resolvida, tornando mais adequada a avaliação de uma série de estruturas como, por exemplo, a coluna vertebral. ${ }^{(17)}$ Acoplada à injeção de contraste intravenoso, tornou possível a realização de exames angiográficos por tomografia (Figura 2).

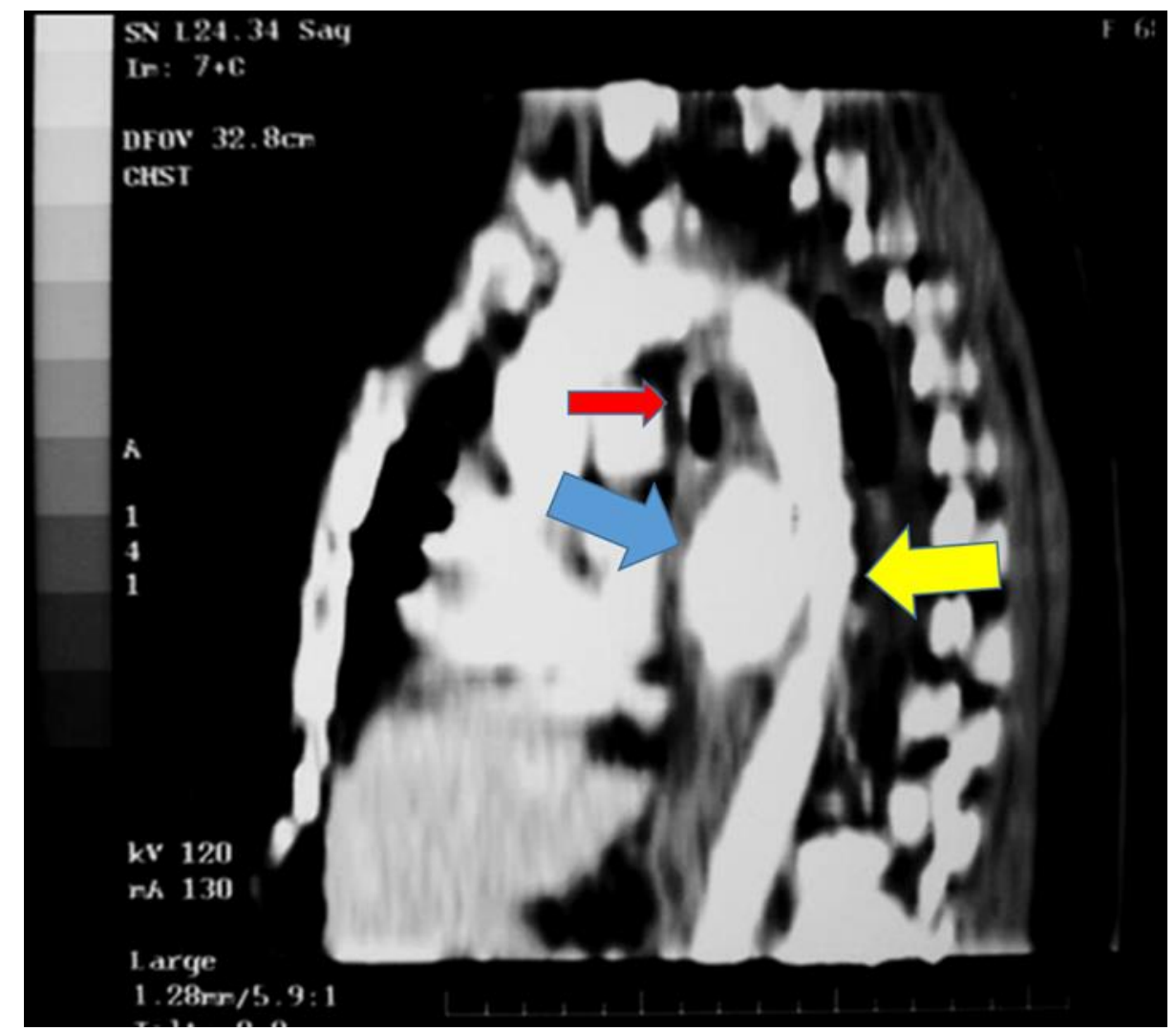

Note a perfuração da parede anterior da aorta torácica com extravasamento de contraste (seta azul), comunicando a aorta com a luz do esôfago.

Figura 2. Reconstrução sagital de angiotomografia realizada em equipamento helicoidal no plano da aorta torácica (seta amarela) e esôfago (seta vermelha).

Um outro salto tecnológico foi incorporado aos equipamentos helicoidais permitindo a obtenção de imagens com muito mais resolução no eixo longitudinal do paciente (eixo z). Foi a era dos equipamentos de múltiplas camadas de detectores (Figura 3). ${ }^{(18)}$ A construção destes tomógrafos é semelhante à do helicoidal, adicionando o uso de múltiplos detectores enfileirados. Os conceitos permanecem os 
mesmos dos idealizados por Hounsfield, entretanto, uma série de melhorias foi incorporada, tornando a tomografia uma poderosa ferramenta diagnóstica. A melhoria da qualidade dos detectores, que passaram a ter melhor relação sinal/ruído e menor espessura (atingindo $0,5 \mathrm{~mm}$ ), a digitalização mais eficiente do sinal, além da miniaturização dos circuitos eletrônicos, a melhoria da qualidade da fonte de raios- $\mathrm{X} \mathrm{e}$ o crescimento exponencial da velocidade computacional são alguns dos exemplos de tecnologias que permitiram expandir as indicações da tomografia para uso diagnóstico, sendo que os estudos vasculares, em particular, foram muito beneficiados.

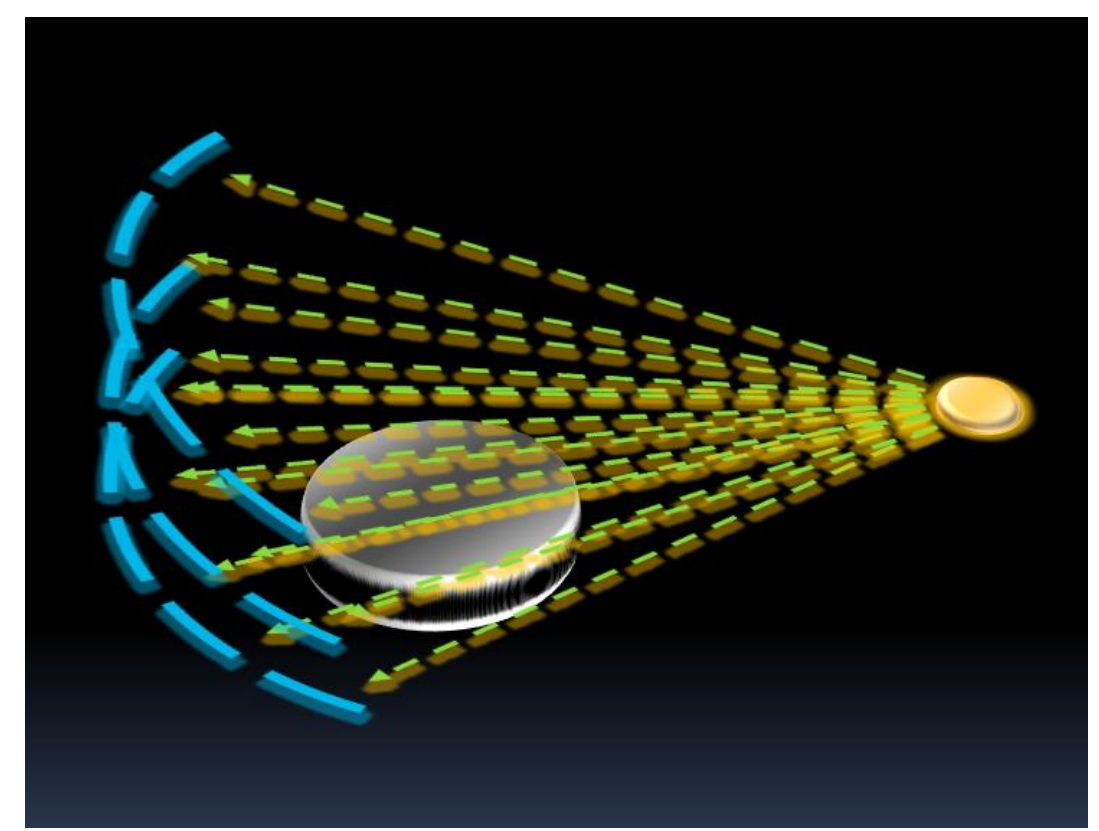

Fonte: Adaptado de Heijenbrok-Kal MH, Kock MC, Hunink MG. Lower extremity arterial disease: multidetector CT angiography metaanalysis. Radiology. 2007;245(2):433-9. ${ }^{(3)}$

Figura 3. Esquema representando um equipamento de tomografia computadorizada de múltiplos detectores

\subsection{Fisiologia e farmacocinética do contraste iodado e sua aplicação em angiotomografia}

Para se realizar qualquer estudo de CTA, é imprescindível o estudo da fisiologia da progressão do contraste no sistema arterial. ${ }^{(7)}$ Este conhecimento deve ser aplicado em combinação com as capacidades técnicas em constante evolução dos equipamentos de tomografia para se obter estudos diagnósticos de qualidade. Inicialmente, estudos utilizando porcos como modelo e extrapolações matemáticas 
contribuíram para o conhecimento da cinética do contraste e foram imprescindíveis para a sua aplicação prática nos exames diagnósticos. ${ }^{(19,20)}$

Após a injeção venosa, o contraste iodado chega ao coração e circula pelas câmaras cardíacas direitas até ganhar a circulação pulmonar através da artéria pulmonar. Do pulmão, o contraste retorna pelas veias pulmonares às câmaras cardíacas esquerdas e a partir do ventrículo esquerdo, ganha a circulação arterial através da aorta.

Algumas considerações merecem destaque neste caminho percorrido pelo contraste iodado. A concentração do contraste que percorre a veia na qual foi injetado depende principalmente de dois fatores: da apresentação do contraste (medida em $\mathrm{mg}$ de lodo $/ \mathrm{mL}$ ) e do fluxo da injeção. $\mathrm{O}$ acesso venoso mais comum é uma veia antecubital e, neste caso, o contraste infundido passa por algumas diluições antes de atingir o átrio direito, nos pontos de confluência com outras veias que estão preenchidas por sangue não contrastado (sendo as principais as jugulares e a veia braquiocefálica contralateral). Outra diluição significativa ocorre no átrio direito, onde o contraste encontra sangue não contrastado da veia cava inferior. Em termos de diluição, a injeção no membro superior direito ou esquerdo não interfere significativamente na contrastação. Entretanto, para os estudos angiográficos, protocola-se a injeção do lado direito para que se evitem artefatos produzidos por contraste em alta concentração ao transitar anteriormente à croça da aorta e seus ramos emergentes no seu trajeto pela luz da veia braquiocefálica esquerda. Caso a injeção venosa ocorra em um dos membros inferiores, o que é pouco usual, os locais de diluição são mais numerosos, pois além das mesmas diluições citadas para a injeção no membro superior, ocorrem diluições significativas na confluência das veias ilíacas comuns, na drenagem das veias renais e nas veias hepáticas. Estas diluições contribuem para reduzir a atenuação vascular arterial no estudo de CTA.

Monitorando-se a contrastação arterial após a injeção do contraste, observa-se que ocorre elevação rápida da atenuação intravascular arterial, seguida de rápida diminuição dessa atenuação. Pouco tempo a seguir, existe novo aumento da atenuação, decorrente do fenômeno de recirculação. ${ }^{(20,21)}$ No caso de injeções longas (em geral, maiores que 15 segundos), essa recirculação potencializa o realce arterial. A recirculação permite que o contraste retorne pelo sistema venoso e se some ao contraste injetado, reduzindo o efeito da diluição, conforme demonstrado na figura 4. Além do aumento da atenuação vascular, outra vantagem da injeção prolongada do 
contraste é de se manter uma atenuação elevada no interior do vaso por maior período, fornecendo "janela temporal" maior para a realização do estudo.

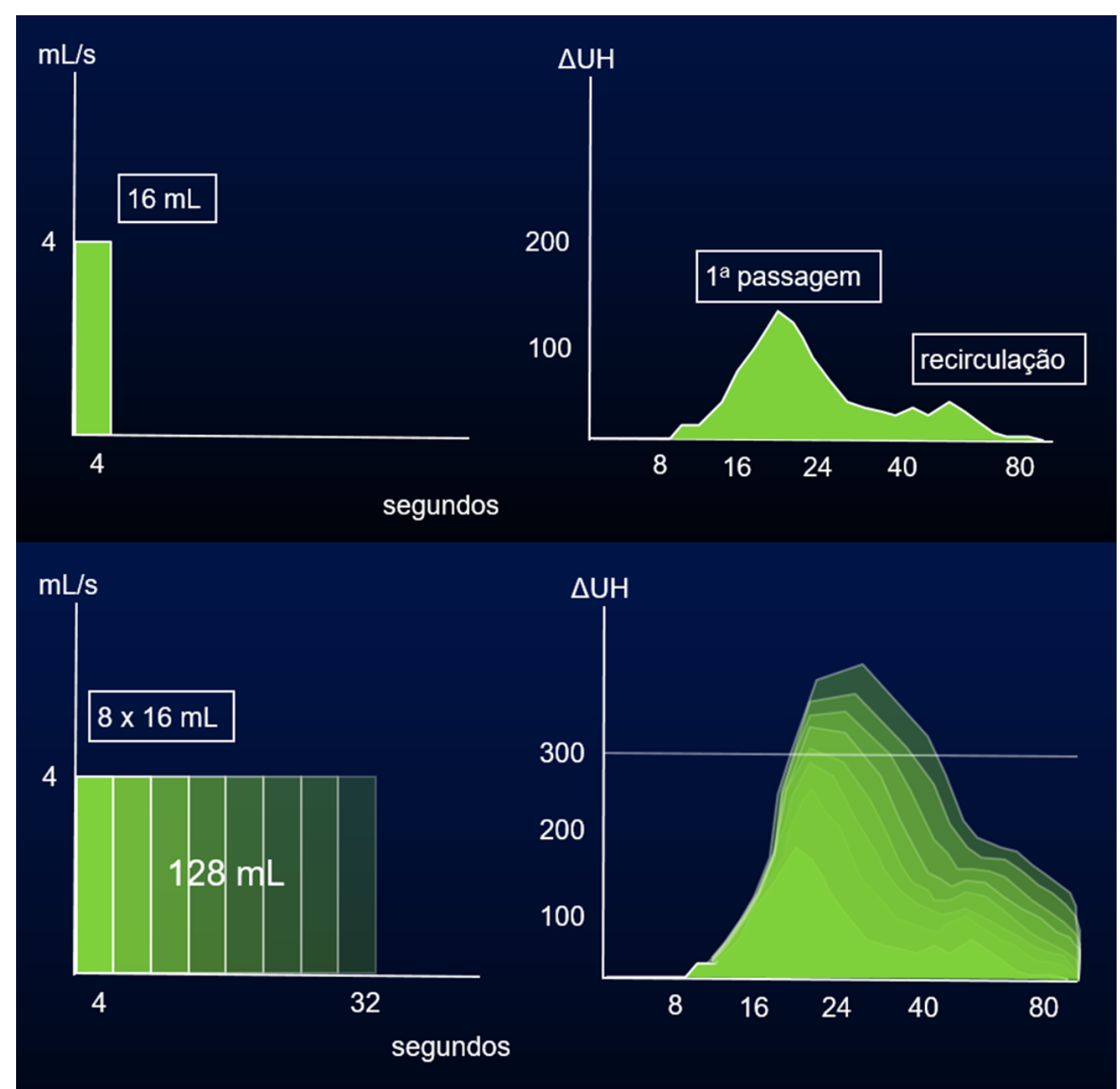

Na parte superior do gráfico a representação de injeção "curta" de 4 segundos e na parte inferior, injeção "longa" de 32 segundos (correspondendo a oito injeções "curtas" consecutivas). A linha horizontal em 300UH é a referência para atenuação diagnóstica.

Fonte: Adaptado de Fleischmann D, Rubin GD. Quantification of intravenously administered contrast medium transit through the peripheral arteries: implications for CT angiography. Radiology. 2005;236(3):1076-82. ${ }^{(7)}$

Figura 4. Variação da atenuação vascular $(\Delta \mathrm{UH})$ durante aquisição angiotomográfica arterial em dois tipos de injeção

Entretanto, existem limitações ao uso do protocolo de injeção prolongada. A principal limitação é a do volume total de contraste a ser administrado (em um adulto médio de $70 \mathrm{~kg}$ e função renal normal, a recomendação é que não se ultrapasse $300 \mathrm{~mL}$ de contraste com concentração de $300 \mathrm{mg} / \mathrm{mL}$, ${ }^{(22)}$ mas na prática, a injeção usual não deve ultrapassar $2 \mathrm{~mL} / \mathrm{kg}$ ). Portanto, devemos utilizar técnicas de injeção que otimizem o contraste como a injeção bifásica (Figura 5). Este tipo de injeção otimiza o contraste, pois determina aumento rápido da atenuação vascular 
devido à primeira fase de injeção com maior fluxo e mantém a alta atenuação com um fluxo menor do contraste na segunda fase.

Utilizando-se este mesmo princípio, porém de forma mais elaborada, foi proposta a técnica de injeção multifásica, em que a variação do fluxo de injeção se ajusta para que se obtenha a contrastação mais homogênea possível. Entretanto, a utilização desta técnica está limitada pela disponibilidade de injetoras automatizadas com esta funcionalidade.

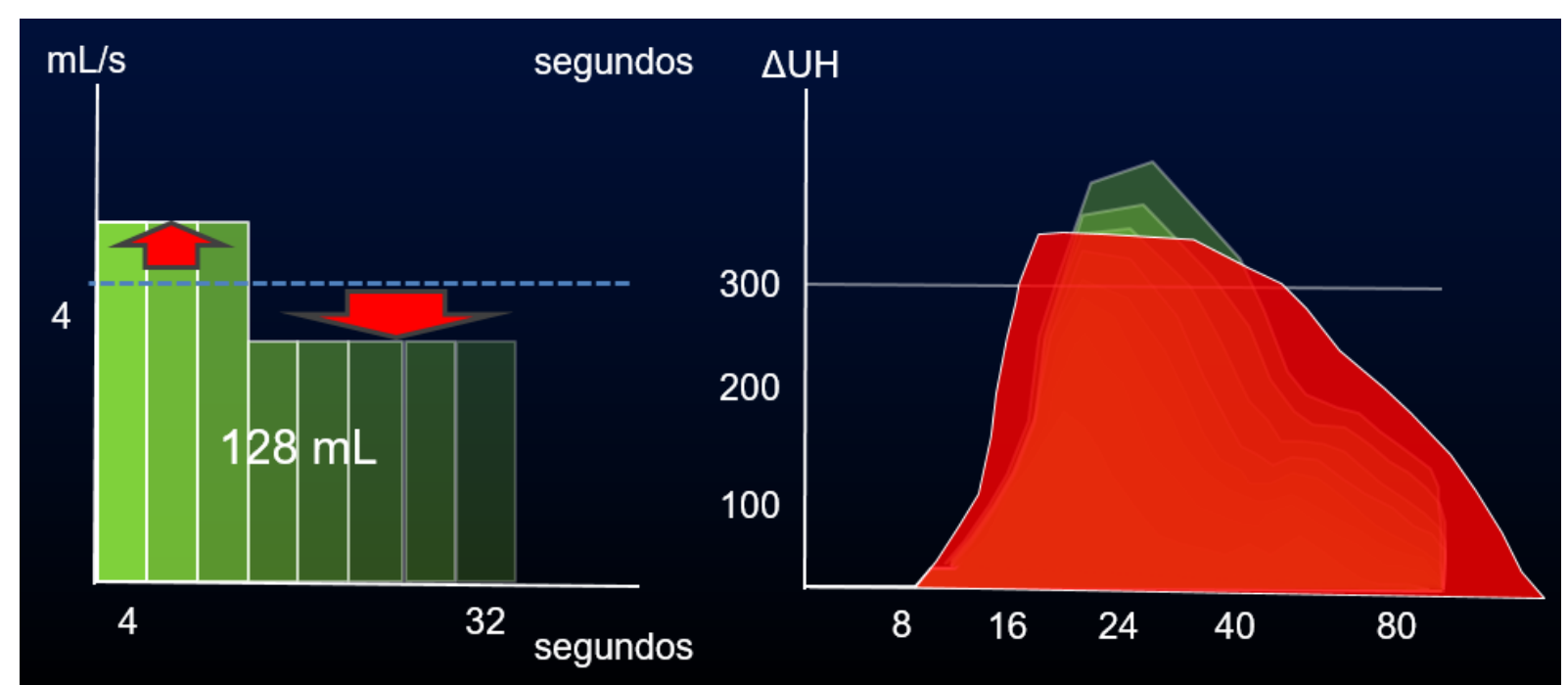

A linha horizontal em 300 UH é a referência para atenuação diagnóstica.

Fonte: Adaptado de Fleischmann D, Rubin GD. Quantification of intravenously administered contrast medium transit through the peripheral arteries: implications for CT angiography. Radiology. 2005;236(3):1076-82. ${ }^{(7)}$

Figura 5. Variação da atenuação vascular $(\Delta U H)$ e o efeito da injeção bifásica (com dois fluxos diferentes de injeção)

2.3 A construção de protocolos otimizados para angiografia por tomografia computadorizada da aorta abdominal, artérias ilíacas e artérias dos membros inferiores com a utilização dos conhecimentos técnicos da fisiologia e farmacocinética do contraste e ajustes técnicos do equipamento

A construção de protocolos de aquisição em CTA da aorta abdominal, artérias ilíacas e artérias dos membros inferiores foi um desafio devido à grande variabilidade de progressão do contraste pelo sistema arterial entre os indivíduos. A variação do pico de contrastação medido na aorta abdominal é de 14 a 32 segundos após o início da injeção, de acordo com estudo de Fleischmann et al. ${ }^{(7)}$ Ainda 
no mesmo estudo, constatou-se que a variação entre o tempo de trânsito entre o pico de contrastação na aorta abdominal e poplítea é de 4 a 24 segundos.

O desenvolvimento desta técnica de exame foi possível utilizando-se os conhecimentos da fisiologia e farmacocinética do contraste, sendo proposta uma injeção "longa" de contraste que pudesse resolver o problema da variabilidade entre pacientes. Cálculos balanceando fluxo de injeção, volume de contraste e o tempo de injeção necessário chegaram à conclusão de que uma injeção de 35 segundos seria suficiente para se realizar com segurança o exame da grande maioria dos pacientes. ${ }^{(7)}$

Historicamente, estes protocolos tiveram que se ajustar à evolução tecnológica dos equipamentos de tomografia, inicialmente sendo separados em dois grupos: aquisições "lentas" (geralmente utilizados em aparelhos com menos de 16 fileiras de detectores) e aquisições "rápidas" (possível apenas em aparelhos com 16 fileiras de detectores ou mais, até 64 fileiras). ${ }^{(23)}$ Atualmente, os protocolos padrão utilizam apenas a aquisição "lenta", devido à maior abrangência desta técnica em relação aos equipamentos disponíveis. Este protocolo "lento", amplamente aceito, é chamado de protocolo "padrão" ao longo desta tese. 


\section{MÉTODOS}

Os pacientes foram informados de todos os aspectos dos procedimentos envolvidos e o consentimento informado foi obtido de todos os participantes. Os comitês institucionais e nacionais de ética em pesquisa aprovaram nosso estudo sob número CAAE 12336513.3.0000.0071.

Este foi um estudo prospectivo. Os pacientes foram recrutados por cirurgiões vasculares e tinham indicação clínica para realizar CTA. Os critérios de inclusão eram indivíduos de pelo menos 12 anos e que pesavam 90kg ou menos, com o intuito de incluir pacientes de tamanho similar. Os critérios de exclusão eram pacientes com contraindicações para uso de contraste iodado intravenoso (alergia prévia grave, insuficiência renal) e gravidez. Um questionário específico foi utilizado para obtenção de dados clínicos e para se obter dados relacionados à segurança do paciente para realização do exame (Anexo 1). A creatinina sérica foi medida em todos os pacientes incluídos (Reflotron, Roche Diagnostics, Rotkreuz, Suíça). A taxa de filtração glomerular abaixo de $30 \mathrm{~mL} / \mathrm{min}$ calculada pela fórmula ${ }^{(24)}$ de Cockroft-Gault foi considerada contraindicação para a injeção de contraste.

Algumas condições específicas foram abordadas de acordo com o protocolo institucional para a injeção de contraste iodado, que incluiu o uso de premedicação para risco moderado a baixo de reação alérgica e hidratação prévia para pacientes com Taxa de Filtração Glomerular estimada entre 30 e 60mL/min.

A pedido de nosso Comitê de Ética institucional, realizamos um estudo inicial de três pacientes antes de iniciar a randomização do grupo usando o novo protocolo proposto. Dois radiologistas (12 e 10 anos de experiência em imagens vasculares) analisaram estes três pacientes cegamente em relação aos métodos de aquisição, pareados com três exames passados escolhidos aleatoriamente em que foi utilizada técnica padrão.

Trinta pacientes foram então arrolados e prospectivamente estudados após terem sido randomizados em dois grupos, sendo que um grupo foi submetido ao novo protocolo testado e o outro grupo foi submetido ao estudo com o protoloco institucional padronizado. Foi utilizada uma ferramenta de pesquisa chamada Randomizer (www.randomizer.org). Uma pessoa independente sem relação com o 
estudo realizou a randomização e o grupo do protocolo do paciente foi mantido em sigilo até o momento do exame.

\subsection{Aquisição de imagem}

Todas as imagens foram adquiridas usando um tomógrafo de 320 fileiras de detectores (Aquilion One, Toshiba Medical Systems, Japão), capaz de operar em modo helicoidal de 64 fileiras ou 160 fileiras.

Os pacientes receberam contraste iodado intravenoso com a mesma concentração (iobitridol, 350mgL/L, Henetix, Guerbet, França) por uma veia antecubital no membro superior direito, através de um cateter de calibre 18 ou 20 e administrado através de uma injetora automática (Stellant Dual Syringe CT, Medrad, Indianola, PA, EUA).

Todos os exames avaliaram a aorta abdominal, artérias ilíacas e as artérias dos membros inferiores. Uma fase sem contraste foi realizada do abdome superior, utilizando um protocolo de dose baixa (modulação automática da dose definida como baixa dose). As imagens sem contraste foram utilizadas para localizar a origem das artérias renais e neste nível, foi colocada uma medida de região de interesse (ROI - Region of Interest) na aorta abdominal para disparar automaticamente a aquisição angiográfica.

Dois biomédicos se alternaram na realização de todos os exames com a técnica descrita acima.

Os pacientes do Grupo 1 foram submetidos à CTA com o novo protocolo de imagem usando aquisição de alta velocidade com 160 fileiras de detectores e fluxo trifásico de injeção de contraste iodado intravenoso, com tempo de varredura de aproximadamente 9 segundos (Tabela 1).

Tabela 1. Parâmetros do protocolo para aquisição de alta velocidade com 160 fileiras de detectores (Grupo 1) e padrão com 64 fileiras de detectores (Grupo 2)

\begin{tabular}{lcc}
\hline & Alta velocidade & Padrão \\
\hline Configuração do detector & $160 \times 0,5 \mathrm{~mm}$ & $64 \times 0,5 \mathrm{~mm}$ \\
Modulação automática de dose & Padrão & Padrão \\
Pitch / Pitch helicoidal & $0,828 / 132,5$ & $0,828 / 53$ \\
Tempo de rotação & $0,35 \mathrm{~s}$ & $0,5-0,75 \mathrm{~s}$ \\
Atenuação de disparo & $170 \mathrm{UH}$ & $170 \mathrm{UH}$ \\
kVp & 100 & 100 \\
Espessura de corte / incremento & $1,0 / 0,8 \mathrm{~mm}$ & $1,0 / 0,8 \mathrm{~mm}$ \\
\hline
\end{tabular}


O protocolo de administração de contraste iodado intravenoso está descrito na tabela 2. Utilizamos a injeção trifásica para manter o contraste homogêneo nas artérias estudadas durante um período de tempo suficientemente longo. Essa abordagem foi necessária para solucionar a variabilidade entre pacientes durante uma aquisição tomográfica de alta velocidade na qual é necessário contrastar um segmento arterial relativamente longo que será estudado quase que instantaneamente. Esse princípio é semelhante à angiografia digital em que todo o segmento arterial estudado deve estar preenchido pelo contraste no ato da aquisição radiográfica. $O$ volume da injeção trifásica foi dividido em $17 \%$ do total de contraste injetado na primeira fase, $66 \%$ na segunda fase e $17 \%$ na terceira fase, sendo que o volume total de contraste administrado depende do peso do paciente.

Tabela 2. Protocolo de injeção trifásica para aquisição de alta velocidade com 160 fileiras de detectores (Grupo 1)

\begin{tabular}{lc}
\hline Peso & Volume de contraste (fluxo da injeção) \\
\hline$<55 \mathrm{~kg}$ & $20 \mathrm{~mL}(4,0 \mathrm{~mL} / \mathrm{s})+80 \mathrm{~mL}(3,2 \mathrm{~mL} / \mathrm{s})+20 \mathrm{~mL}(2,5 \mathrm{~mL} / \mathrm{s})$ \\
$56-65 \mathrm{~kg}$ & $23 \mathrm{~mL}(4,5 \mathrm{~mL} / \mathrm{s})+90 \mathrm{~mL}(3,6 \mathrm{~mL} / \mathrm{s})+23 \mathrm{~mL}(3,0 \mathrm{~mL} / \mathrm{s})$ \\
$66-85 \mathrm{~kg}$ & $25 \mathrm{~mL}(5,0 \mathrm{~mL} / \mathrm{s})+100 \mathrm{~mL}(4,0 \mathrm{~mL} / \mathrm{s})+25 \mathrm{~mL}(3,2 \mathrm{~mL} / \mathrm{s})$ \\
$86-90 \mathrm{~kg}$ & $28 \mathrm{~mL}(5,5 \mathrm{~mL} / \mathrm{s})+110 \mathrm{~mL}(4,4 \mathrm{~mL} / \mathrm{s})+28 \mathrm{~mL}(3,7 \mathrm{~mL} / \mathrm{s})$ \\
\hline
\end{tabular}

Os exames do Grupo 2 foram realizados com nosso protocolo institucional padrão, com base em estudos de Fleischmann et al. ${ }^{(8)}$ (Tabela 1). 0 tempo de varredura total para o protocolo CTA do Grupo 2 foi de aproximadamente 40 segundos.

A administração de contraste intravenoso para o Grupo 2 seguiu o protocolo padrão de injeção bifásica e seguiu os parâmetros especificados na tabela 3.

Tabela 3. Protocolo de injeção bifásica para aquisição padrão de 64 fileiras de detectores (Grupo 2)

\begin{tabular}{lc}
\hline Peso & Volume de contraste (fluxo de injeção) \\
\hline$<55 \mathrm{~kg}$ & $20 \mathrm{~mL}(4,0 \mathrm{~mL} / \mathrm{seg})+96 \mathrm{~mL}(3,2 \mathrm{~mL} / \mathrm{s})$ \\
$56-65 \mathrm{~kg}$ & $23 \mathrm{~mL}(4,5 \mathrm{~mL} / \mathrm{seg})+108 \mathrm{~mL}(3,6 \mathrm{~mL} / \mathrm{s})$ \\
$66-85 \mathrm{~kg}$ & $25 \mathrm{~mL}(5,0 \mathrm{~mL} / \mathrm{seg})+120 \mathrm{~mL}(4,0 \mathrm{~mL} / \mathrm{s})$ \\
$86-90 \mathrm{~kg}$ & $28 \mathrm{~mL}(5,5 \mathrm{~mL} / \mathrm{seg})+132 \mathrm{~mL}(4,4 \mathrm{~mL} / \mathrm{s})$ \\
\hline
\end{tabular}

\subsection{Pós-processamento}

A série de imagens obtidas com $1,0 \mathrm{~mm}$ de espessura e $0,8 \mathrm{~cm}$ de incremento foram reconstruídas para se obter imagens tridimensionais. Os mesmos 
dois biomédicos que realizaram os exames foram os responsáveis pelas reconstruções, que incluíram vinte vistas de cada segmento (abdômen/pelve, coxas e pernas) com as técnicas de renderização de volume (3D VR) e as mesmas visualizações na projeção de intensidade máxima (3D MIP), demonstradas nas figuras 6 e 7, respectivamente.

As imagens foram enviadas para um sistema de comunicação e arquivo de imagens (PACS Carestream Health, Rochester, Nova lorque, EUA) para análise. 


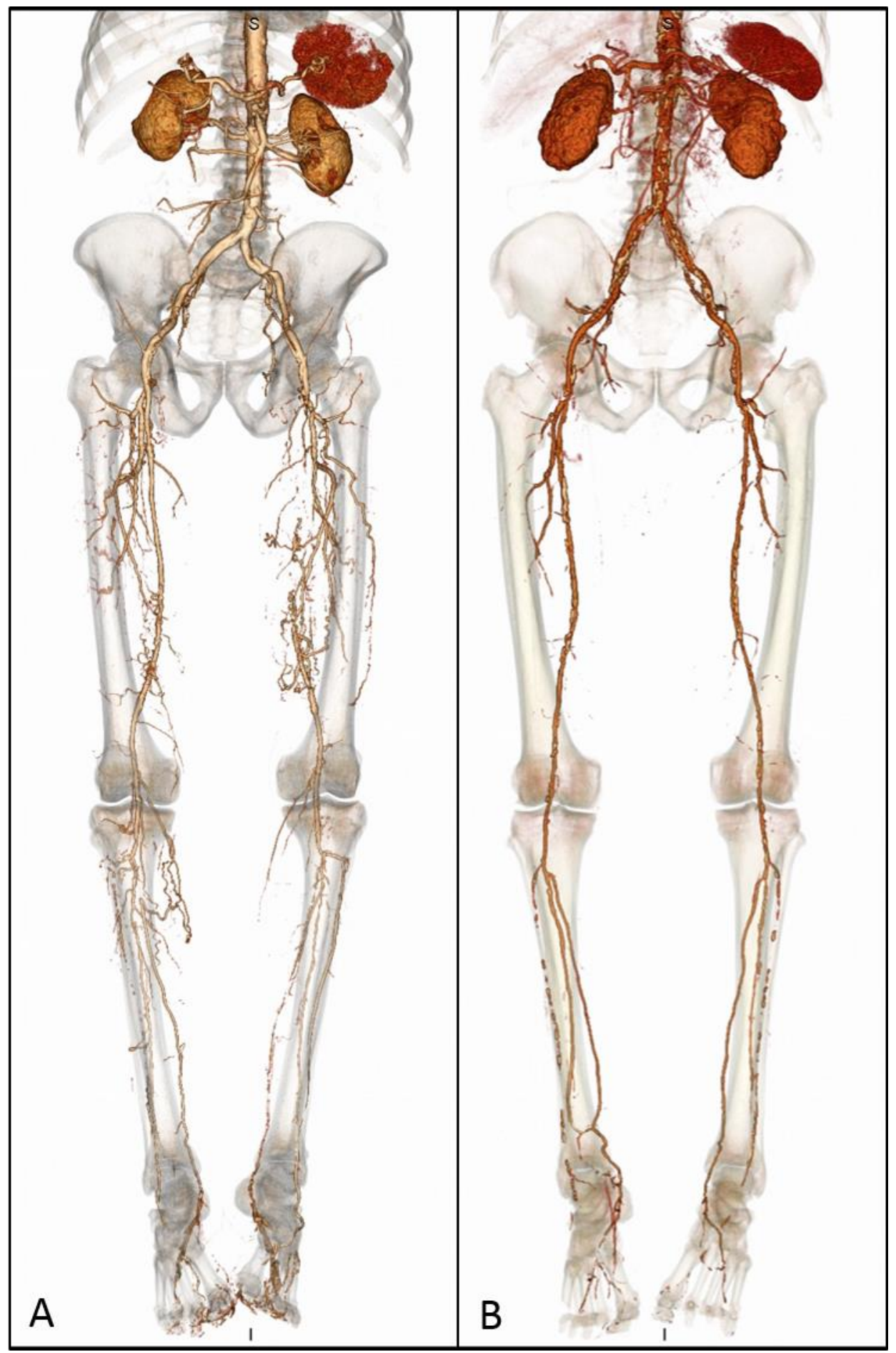

Figura 6. Reconstruções tridimensionais em renderização volumétrica de angiografia por tomografia de paciente estudado com protocolo padrão $(A)$ e outro paciente avaliado com protocolo de alta velocidade de aquisição (B) 


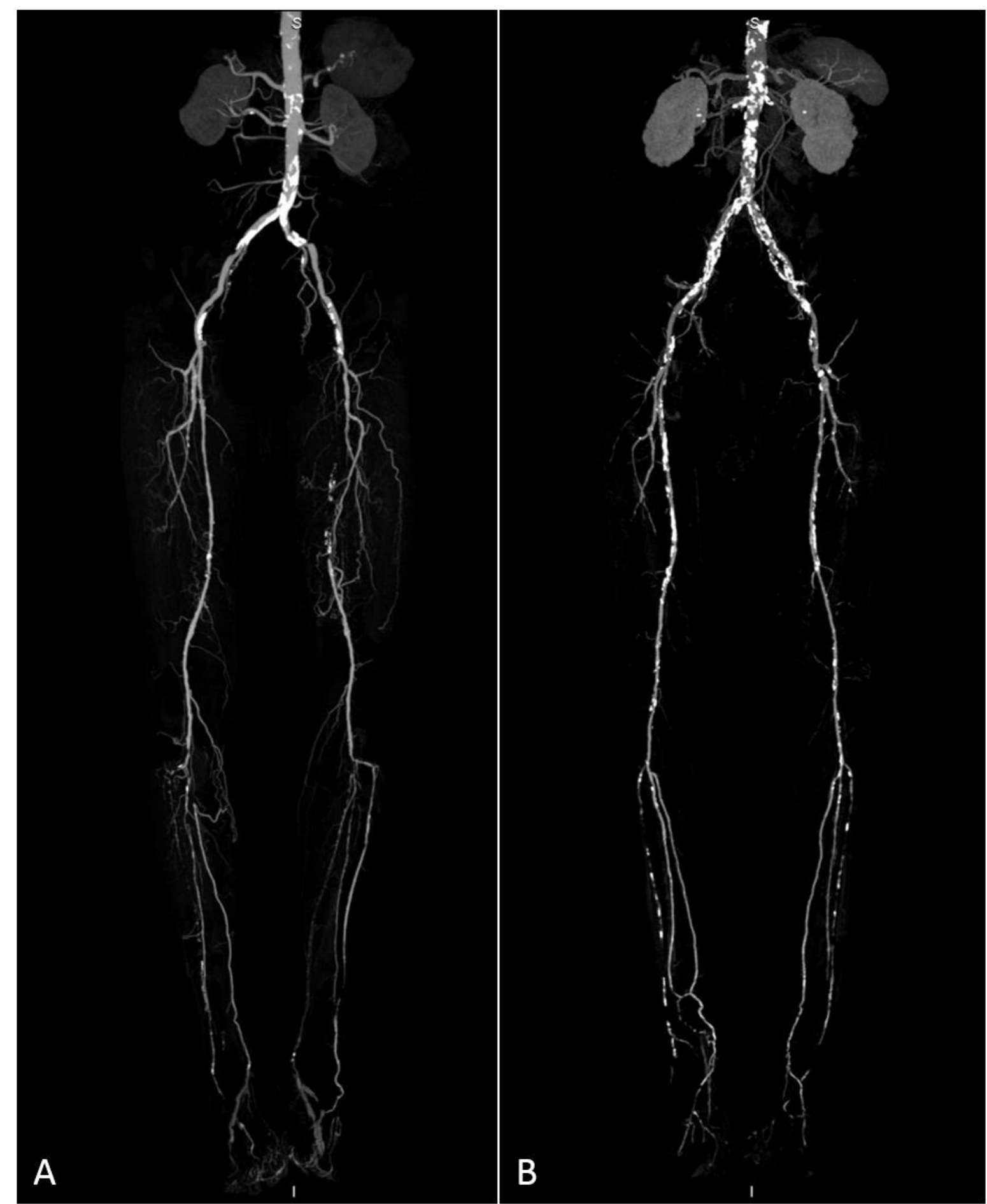

Figura 7. Reconstruções em projeção de intensidade máxima de angiografia por tomografia dos mesmos pacientes da figura 6 , estudados com protocolo padrão (A) e outro avaliado com protocolo de alta velocidade de aquisição $(B)$

\subsection{Análise de imagem}

Os exames anonimizados foram analisados por um radiologista experiente e um cirurgião vascular (ambos com 12 anos de experiência com imagem 
vascular) em estações de trabalho dedicadas usando Carestream PACS v11 (Carestream Health, Rochester, NY, EUA) e monitores de 6 megapixels e 30 polegadas.

A análise de qualidade foi realizada utilizando uma escala de quatro pontos (Quadro 1) e uma nota foi atribuída para cada segmento: aorta abdominal, cada lado das artérias ilíacas comuns, femorais comuns, femorais superficiais, poplíteas e artérias das pernas. No caso das artérias das pernas, um único valor foi atribuído para o conjunto das três artérias (artérias tibiais anteriores, posteriores fibulares). Da mesma forma, as artérias dos pés (pediosa e plantares) receberam um único valor atribuído para o cômputo geral da contrastação. A mesma escala foi utilizada para avaliar as imagens 3D pós-processadas (uma nota para cada paciente).

Quadro 1. Escala de quatro pontos para análise qualitativa

1. Não diagnóstico: informações diagnósticas pobres, impossível detectar ou excluir lesões vasculares.

2. Qualidade diagnóstica moderada: contrastação vascular inomogênea na luz do vaso, avaliação de possíveis lesões vasculares com baixa confiança diagnóstica.

3. Boa visibilização: contrastação na luz do vaso boa e quase totalmente homogênea, avaliação de possíveis lesões vasculares com confiança diagnóstica satisfatória.

4. Excelente visibilização: contrastação na luz do vaso ótima e completamente homogênea, avaliação de lesões vasculares com alta confiança diagnóstica.

Fonte: Adaptado de lezzi R, Santoro M, Marano R, Di Stasi C, Dattesi R, Kirchin M, et al. Low-dose multidetector CT angiography in the evaluation of infrarenal aorta and peripheral arterial occlusive disease. Radiology. 2012;263(1):287-98..$^{(25)}$

A análise objetiva consistiu em medir a atenuação média em Unidades Hounsfield (UH) de uma ROI. O ROI foi colocado em uma posição central do vaso e incluiu pelo menos dois terços do seu lúmen. Os segmentos analisados foram: aorta abdominal (ao nível das artérias renais), artérias femorais comuns (terço médio), artérias femorais superficiais (terço médio) e artérias poplíteas (no nível do platô tibial).

Além disso, o ruído na imagem foi medido como o desvio padrão da média da atenuação e foi calculado colocando um ROI no músculo iliopsoas no nível da quinta vértebra lombar (L5) e um segundo $\mathrm{ROI}$ no gastrocnêmio medial da perna esquerda. As medições foram realizadas por dois tecnólogos experientes (8 e 5 anos de experiência), e a técnica foi validada por meio de testes em um conjunto de três exames de pacientes recuperados de nosso arquivo digital.

A dose de radiação também foi comparada entre todos os exames para identificar seus efeitos na qualidade da imagem e foi obtida a partir do relatório de dose fornecido automaticamente pelo scanner no final de cada exame. A medida 
comparada foi o produto dose-comprimento (DLP - Dose Lenght Product), expresso em mGy.cm.

\subsection{Análise estatística}

As variáveis contínuas foram expressas como média e desvio padrão e comparadas usando o teste $t$ de Student para distribuições normais. Para distribuições não normais, as variáveis contínuas foram expressas como mediana e intervalo interquartil e comparadas utilizando o teste de Mann-Whitney, que também foi usado na comparação de variáveis ordinais qualitativas. As variáveis categóricas foram descritas por tabelas de frequências e porcentagens e os grupos foram comparados pelo teste exato de Fischer. A normalidade das variáveis contínuas foi verificada visualmente por histograma, boxplot e gráficos Quantile-Quantile.

A concordância entre observadores foi avaliada utilizando o coeficiente de concordância de Kendall (W - adequado para categorias ordinais).

Para avaliações de qualidade objetivas, analisamos as médias obtidas pelas duas medidas.

Equações de estimativa generalizada com matriz de correlação não estruturada assumindo a distribuição marginal normal ou a distribuição de Poisson e a função de ligação de identidade foram usadas para comparar os dois grupos, sendo utilizados os dados obtidos de cada segmento separadamente. As análises foram realizadas em $\mathrm{R}$ v3.0.3. 


\section{RESULTADOS}

\subsection{Dados demográficos}

Foram incluídos 30 indivíduos recomendados para avaliação da doença arterial oclusiva periférica, 16 mulheres e 14 homens, com faixa etária média de 63,4 anos (intervalo entre 20 e 87 anos). As características dos dois grupos foram semelhantes e não houve diferença estatística na idade, gênero e peso $(p>0,05)$. As características da população do estudo são descritas na tabela 4.

Tabela 4. Dados demográficos

\begin{tabular}{lccc}
\hline Parâmetro & $\begin{array}{c}\text { Grupo 1 (Aquisição de } \\
\text { alta velocidade, } \mathbf{n = 1 5}\end{array}$ & $\begin{array}{c}\text { Grupo 2 (Aquisição } \\
\text { padrão, } \mathbf{n = 1 5 )}\end{array}$ & Valor-p \\
\hline Idade, média (dp), anos & $60,9(16,5)$ & $65,9(14,8)$ & 0,385 \\
Peso, média (dp), $\mathrm{kg}$ & $66,7(13,1)$ & $66,9(12,3)$ & 0,966 \\
IMC, média (dp), $\mathrm{kg} / \mathrm{m}^{2}$ & $25,2(1,1)$ & $25,3(1,2)$ & 0,79 \\
\hline
\end{tabular}

dp: desvio padrão.

\subsection{Volume de contraste e tempo de aquisição das imagens}

A mediana do volume de contraste utilizado para os exames foi de 136mL (intervalo total de 116 - 160mL). Para o Grupo 1 (aquisição rápida), a mediana do volume administrado foi $136 \mathrm{~mL}$ (intervalo total de 120 a $150 \mathrm{~mL}$ ) e para o Grupo 2, 145mL (intervalo total de 116 - 160mL). O protocolo de injeção multifásica para o grupo de aquisição rápida prevê 3,4 a 3,7\% a mais de contraste, dependendo do peso do paciente. Portanto, não foi realizada análise estatística deste parâmetro, uma vez que já existe diferença por padrão técnico.

O tempo de aquisição de imagens, que foi um dos propósitos da nossa análise, foi significativamente diferente entre os grupos. O tempo médio de aquisição para o Grupo 1 foi de 9,1 segundos (desvio padrão de 0,4 segundos) e para o Grupo 2 foi de 38,3 segundos (desvio padrão de 2,4 segundos). 


\subsection{Concordância entre observadores}

A concordância entre os observadores radiologista e cirurgião vascular foi alta $(\mathrm{W}=0,959, \mathrm{p}<0,001)$ para 379 segmentos analisados (excluindo segmentos ocluídos) e 30 reconstruções pós-processadas em 3D. Uma vez que encontramos concordância entre os observadores, a avaliação do radiologista foi escolhida para análise de qualidade subjetiva.

\subsection{Qualidade da imagem}

Foram avaliados 187 segmentos arteriais para os 15 pacientes que compuseram o Grupo 1 e 190 segmentos para os outros 15 pacientes do Grupo 2. A análise dos segmentos usando a escala de quatro pontos não mostrou diferença estatisticamente significativa entre os grupos usando equações de estimação generalizadas. A maioria das imagens foi de qualidade suficientemente alta com pontuações 3 ou 4 (bom ou excelente para o diagnóstico), com valores médios e intervalos de confiança de $95 \%$ de 3,60 (3,39 - 3,81) para o Grupo 1 e 3,62 (3,40 3,83 ) para o Grupo 2. Quando as notas foram agrupadas e categorizadas em diagnóstico ( 3 e 4 ) e não diagnóstico ( 1 e 2), encontramos melhor desempenho para o Grupo 1 (aquisição rápida), com 99,5\% de segmentos com valor diagnóstico. Em contrapartida, 96,4\% dos segmentos foram considerados diagnósticos para o Grupo 2 (aquisição padrão) com valor de p de 0,034.

As medidas objetivas da luz arterial apresentaram menor atenuação para o Grupo 1 (aquisição rápida) utilizando equações de estimação generalizadas para análise estatística. Os valores médios e os intervalos de confiança de $95 \%$ foram de $426,85 \mathrm{UH}(399,99$ - 453,70) para o Grupo 1 e 517,67UH $(590,64$ - 544,70) para o Grupo 2.

\subsection{Dose de radiação e ruído}

Os dados coletados do relatório de dose fornecido pelo equipamento não apresentaram diferença estatisticamente significativa em relação à dose de 
radiação entre os grupos, porém a dose tende a ser maior para o Grupo 1. O DLP médio foi de 1296,99 $\pm 377,44$ e 1082,69 $\pm 279,74$ mGy.cm, respectivamente, para os Grupos 1 e 2 (valor de p de 0,88).

O ruído medido por meio do desvio padrão da atenuação nos tecidos adjacentes às artérias analisadas demonstrou menos ruído no iliopsoas para o grupo de aquisição de alta velocidade (Grupo 1, 20,66 \pm 2,94; Grupo 2, 25,21 \pm 3,21; $p<0,001)$ e nenhuma diferença estatisticamente significativa entre os grupos no gastrocnêmio (Grupo 1, 19,10 \pm 5,83, Grupo 2, 21,72 + 4,03).

Os resultados das análises estão resumidos nas tabelas 5 e 6.

Tabela 5. Resumo dos resultados (análises por paciente)

\begin{tabular}{lccc}
\hline Parâmetro & Grupo 1 & Grupo 2 & Valor-p \\
\hline Volume de contraste $(\mathrm{mL})$ & (Aquisição rápida, $\mathbf{n = 1 5})$ & (Aquisição padrão, $\mathbf{n = 1 5})$ & \\
Tempo de aquisição $(\mathrm{s})$ & $138,8 \mathrm{~mL}(120-150 \mathrm{~mL})$ & $145 \mathrm{~mL}(116-160 \mathrm{~mL})$ & - \\
Dose de radiação $(\mathrm{mGy} . \mathrm{cm})$ & $9,1(8,5-10,1 \mathrm{~s})$ & $38,3(32,4-41,9)$ & $<0,001$ \\
\hline
\end{tabular}

Tabela 6. Resumo dos resultados (análises por segmentos vasculares)

\begin{tabular}{lccc}
\hline Parâmetro & $\begin{array}{c}\text { Grupo 1 } \\
\text { (Aquisição rápida, } \mathbf{n = 1 8 7 )}\end{array}$ & $\begin{array}{c}\text { Grupo 2 } \\
\text { (Aquisição padrão, } \mathbf{n = 1 9 0 )}\end{array}$ & Valor-p \\
\hline Qualidade da imagem & $3,60(3,39-3,81)$ & $3,62(3,40-3,83)$ & 0,64 \\
Realce vascular $(\mathrm{UH})^{\star}$ & $426,85(399,99-453,70)$ & $517,67(590,64-544,70)$ & $<0,001$ \\
Qualidade diagnóstica $(\%)^{*}$ & 99,5 & 96,4 & 0,034 \\
\hline
\end{tabular}




\section{DISCUSSÃO}

Demonstramos que uma aquisição de alta velocidade usando a capacidade completa de um equipamento helicoidal de 160 fileiras de detectores para estudo das artérias dos membros inferiores tem qualidade diagnóstica superior à técnica padrão com o novo protocolo descrito em nosso estudo. Fleischmann e Rubin, em 2005, estudaram o trânsito do meio de contraste através das artérias periféricas dos membros inferiores e propuseram dois protocolos diferentes para CTA: aquisição "lenta" (aproximadamente 40s) e aquisição "rápida" (15-30s). ${ }^{(26)}$ Mais tarde, em 2010 , Fleischmann melhorou este protocolo usando uma injeção bifásica, mas recomendou uma aquisição "lenta". No entanto, as aquisições rápidas podem ser valiosas para reduzir os artefatos de movimento (principalmente da respiração e do movimento involuntário). ${ }^{(27)} \mathrm{O}$ principal desafio para realizar uma aquisição tomográfica "rápida" das artérias dos membros inferiores é manter um realce homogêneo do contraste em todos os segmentos arteriais estudados dentro de uma pequena janela temporal, que está se tornando cada vez mais curta à medida que a tecnologia evolui (aproximadamente 9s como observado em nosso estudo). Bae estudou a injeção de contraste em um modelo porcino (em 2000) e em humanos (2004) concluindo que um protocolo multifásico que usava uma injeção de contraste exponencialmente desacelerada ajustada para o débito cardíaco foi a melhor maneira de realizar o realce vascular prolongado e uniforme em CTA. ${ }^{(12,28)}$

Com base no conhecimento prévio, formulamos um protocolo de injeção multifásica adaptado para nossas injetoras de contraste usando um protocolo trifásico. Nossos resultados mostraram que essa abordagem tem qualidade diagnóstica superior ao protocolo padrão, provavelmente pela técnica ter menor influência da variabilidade entre pacientes devido a efeito minimizado da velocidade de progressão do contraste no sistema arterial, aquisição tomográfica mais tardia e redução dos artefatos de movimentação. Encontramos maior atenuação vascular nas artérias estudadas com o protocolo padrão, o que pode ser explicado pela menor duração da injeção de contraste, levando a um bolo mais compacto e a aquisição tomográfica mais precoce. No entanto, esta maior atenuação não se traduz em maior capacidade de diagnóstico ou melhores reconstruções em 3D. Uma descoberta inesperada foi a tendência de maior dose de radiação para o protocolo de aquisição "rápido", pois mantivemos os mesmos parâmetros de qualidade para ambos os grupos. Além disso, o 
aumento da dose de radiação resultou em menos ruído. Isso talvez seja uma compensação para a aquisição "rápida" em que a modulação automática da dose se torna menos efetiva. Em contrapartida, observou-se menor ruído na região abdominal para este protocolo, traduzindo melhoria na imagem pelo uso de mais radiação.

A principal limitação deste estudo foi a pequena amostra de população $(\mathrm{N}=30)$. No entanto, esse tamanho de amostra foi efetivo para comparar os grupos com nossos objetivos, e julgamos que era representativo da população com indicações clínicas para CTA. Além disso, o projeto prospectivo randomizado ajudou a alcançar grupos demográficos comparáveis. Outra limitação foi o possível viés decorrente do cegamento ineficaz dos avaliadores durante a leitura da imagem. Especialmente o radiologista pode reconhecer o protocolo utilizado apenas pela análise das imagens. No entanto, a concordância entre observadores foi muito alta para o radiologista e o cirurgião vascular, que está menos atento para a técnica de aquisição e suas possíveis diferenças.

Este estudo pretendeu aplicar uma série de conhecimentos existentes e propôs um novo método para o estudo por imagem da circulação arterial da aorta abdominal, artérias ilíacas e artérias dos membros inferiores. Essa descrição é de particular interesse aos serviços de diagnóstico por imagem que tenham à disposição equipamentos de tomografia de última tecnologia, com alta cobertura e que podem permanecer subutilizados por falta de protocolos técnicos que tirem o máximo proveito das vantagens disponíveis. O conhecimento adquirido poderá auxiliar a se obter diagnósticos mais precisos por possibilitar a realização de aquisições mais rápidas e de alta qualidade, possivelmente com menos artefatos, principalmente em pacientes que tenham dificuldades para se manter imóveis durante a aquisição. Outras condições clínicas em que este novo conhecimento pode ser aplicado são casos em que a velocidade de progressão do contraste no sistema arterial está alterada por lesões vasculares como fístulas arteriovenosas e malformações arteriovenosas de alto fluxo.

O atual estudo traz ainda novos questionamentos que poderão ser motivo de investigações adicionais. A relação entre a velocidade de progressão do contraste no sistema arterial e parâmetros de ultrassonografia com Doppler podem ser estudados, inclusive, com a utilização de contraste ultrassonográfico para que se possa obter ajuste prévio do protocolo da angiotomografia e possivelmente reduzir a dose de 
contraste utilizada. Existe oportunidade para estudar e sugerir melhorias na modulação da radiação com aquisição tomográfica de alta velocidade. 


\section{CONCLUSÕES}

1. É possível utilizar o protocolo otimizado proposto para realização de angiografia por tomografia computadorizada com aquisição de alta velocidade em angiotomografia, utilizando a máxima capacidade de equipamento com 160 fileiras de detectores, combinada à injeção multifásica de contraste com volume minimamente maior de contraste;

2. Comprovamos que a nova técnica de aquisição em alta velocidade proporciona maior qualidade diagnóstica, embora determine menor realce vascular;

3. Existe tendência de maior radiação com o uso da nova técnica, porém, resultando em menor ruído nas imagens obtidas. 
7 ANEXOS

Anexo 1. Questionário específico

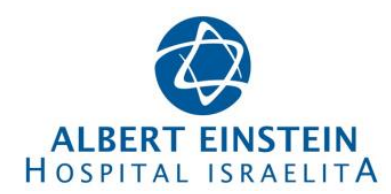

\section{QUESTIONÁRIO DE ENTREVISTA PARA ANGIOTOMOGRAFIA DE AORTA E MEMBROS INFERIORES}

Atenção: Estas perguntas visam orientar a realização de seu exame.

Caso haja dúvidas, elas poderão ser esclarecidas com o biomédico ou médico radiologista, antes de realizar o procedimento.

1. Qual o motivo deste exame? Tente resumir seu problema ou queixa principal.

\begin{tabular}{l}
$\overline{ }$ \\
\hline \\
\hline 2. Sente dores nas pernas? $\quad \square$ Não $\quad \square$ Sim \\
Se sim, há quanto tempo? \\
As dores aparecem em quais situações: \\
$\square$ Ao repouso $\quad \square$ Ao caminhar - Quantos metros? \\
3. Notou algum tipo de alteração nas pernas e/ou pés? \\
$\square$ Não $\quad \square$ Sim \\
$\square$ Inchaço $\quad \square$ Ferimentos \\
\end{tabular}

\section{Já fez alguma cirurgia ou angioplastia (procedimento} vascular)? $\square$ Não $\square$ Sim

Se sim, quais e quando?

$5 . \quad$ Já fez:

$\square$ Quimioterapia: há quanto tempo?

\section{$\square$ Radioterapia: há quanto tempo?}

Já fez ou irá fazer:

$\square$ lodoterapia: Há quanto tempo/quando?

\section{Qual motivo/região do corpo?}

6. Já teve reação por contraste iodado?

$\square$ Não $\square$ Sim Se sim, descreva a reação:

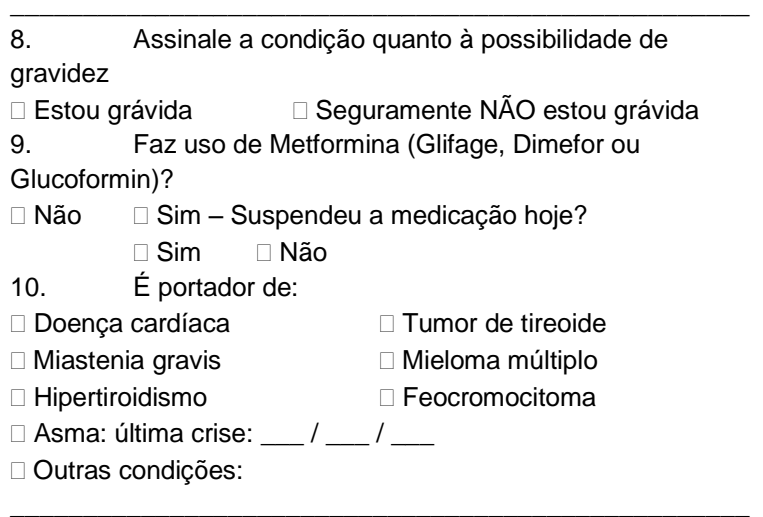

Questionário de Choyke

Alguém já te disse que você tem problema renal?
$\square$ Não
$\square \operatorname{Sim}$

Alguém já te disse que você tem proteína na urina?

$\square$ Não $\quad \square \operatorname{Sim}$

Você tem pressão alta? $\square$ Não $\square$ Sim

Você tem diabetes? $\square$ Não $\square$ Sim

Você tem gota? $\square$ Não $\square$ Sim

Você já fez cirurgia renal? $\square$ Não $\square$ Sim

11. Creatinina:_Ureia:___ Clearance:

12. É:

$\square$ Tabagista - quantos maços por dia?

$\square$ Ex-tabagista - parou há quanto tempo?

Quantos maços e por quanto tempo fumou?

13. Peso: $\frac{\text { Qúltima refeição: }}{\text { 14. }}$ Altura:
14.

\begin{tabular}{l}
\hline 7. Tem alguma alergia? \\
$\square$ Não $\quad \square$ Sim Se sim, a quê?
\end{tabular}

Assinatura do paciente ou responsável

** Preenchimento exclusivo enfermagem/biomédicos **

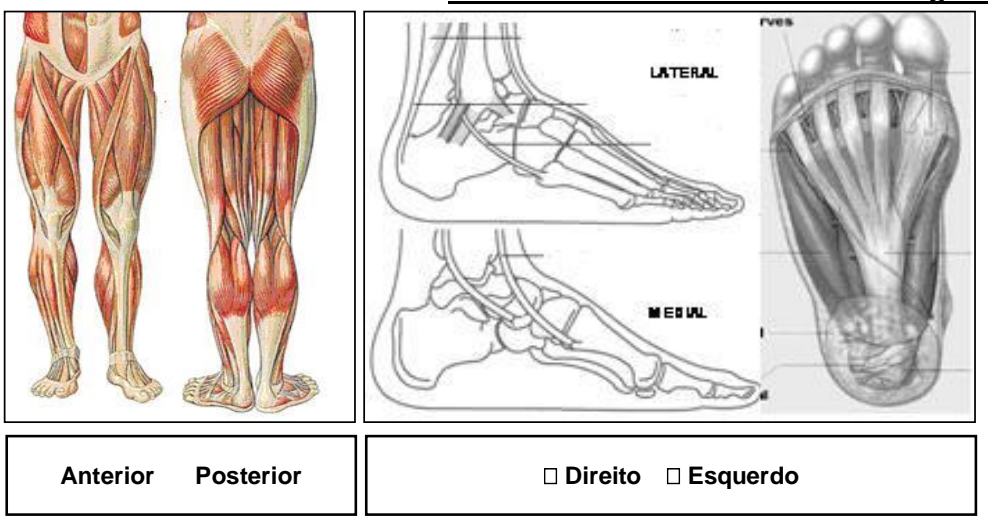

Descrição: 


\section{REFERÊNCIAS}

1. Patel MC, Levin DC, Parker L, Rao VM. Have CT and MR angiography replaced catheter angiography in diagnosing peripheral arterial disease? J Am Coll Radiol. 2015;12(9):909-14.

2. Werncke T, Ringe KI, von Falck C, Kruschewski M, Wacker F, Meyer BC. Diagnostic confidence of run-off CT-angiography as the primary diagnostic imaging modality in patients presenting with acute or chronic peripheral arterial disease. PLoS One. 2015;10(3):e0119900.

3. Heijenbrok-Kal MH, Kock MC, Hunink MG. Lower extremity arterial disease: multidetector CT angiography meta-analysis. Radiology. 2007;245(2):433-9.

4. McGill HC Jr, McMahan CA, Herderick EE, Zieske AW, Malcom GT, Tracy RE, Strong JP; Pathobiological Determinants of Atherosclerosis in Youth (PDAY) Research Group. Obesity accelerates the progression of coronary atherosclerosis in young men. Circulation.

2002;105(23):2712-8.

5. Willmann JK, Baumert B, Schertler T, Wildermuth S, Pfammatter T, Verdun FR, et al. Aortoiliac and lower extremity arteries assessed with 16-detector row CT angiography: prospective comparison with digital subtraction angiography. Radiology. 2005;236(3):1083-93.

6. Bui TD, Gelfand D, Whipple S, Wilson SE, Fujitani RM, Conroy R, et al. Comparison of CT and catheter arteriography for evaluation of peripheral arterial disease. Vasc Endovascular Surg. 2005;39(6):481-90.

7. Fleischmann D, Rubin GD. Quantification of intravenously administered contrast medium transit through the peripheral arteries: implications for CT angiography. Radiology.

2005;236(3):1076-82.

8. Fleischmann D. CT angiography: injection and acquisition technique. Radiol Clin North Am. 2010;48(2):237-47.

9. Meyer BC, Oldenburg A, Frericks BB, Ribbe C, Hopfenmüller W, Wolf KJ, et al. Quantitative and qualitative evaluation of the influence of different table feeds on visualization of peripheral arteries in CT angiography of aortoiliac and lower extremity arteries. Eur Radiol.

2008;18(8):1546-55.

10. Barrett JF, Keat N. Artifacts in CT: recognition and avoidance. Radiographics.

2004;24(6):1679-91.

11. McNitt-Gray MF. AAPM/RSNA physics tutorial for residents: topics in CT. Radiation dose in CT. Radiographics. 2002;22(6):1541-53.

12. Bae KT, Tran HQ, Heiken JP. Uniform vascular contrast enhancement and reduced contrast medium volume achieved by using exponentially decelerated contrast material injection method. Radiology. 2004;231(3):732-6.

13. Ginat DT, Gupta R. Advances in computed tomography imaging technology. Annu Rev Biomed Eng. 2014;16:431-53.

14. Beckmann EC. CT scanning the early days. Br J Radiol. 2006;79(937):5-8.

15. Hounsfield GN. Computed medical imaging. 1979 [cited 2017 Sep 3]. Available from: https://www.nobelprize.org/nobel_prizes/medicine/laureates/1979/hounsfield-lecture.pdf 
16. Cormack AM. Early two-dimensional reconstruction and recent topics stemming from it. 1979 [cited 2017 Sep 3]. Available from:

https://www.nobelprize.org/nobel_prizes/medicine/laureates/1979/cormack-lecture.pdf

17. Mahesh M. Search for isotropic resolution in CT from conventional through multiple-row detector. Radiographics. 2002 Jul-Aug;22(4):949-62. Review.

18. Kalra MK, Maher MM, D'Souza R, Saini S. Multidetector computed tomography technology: current status and emerging developments. J Comput Assist Tomogr. 2004;28 Suppl 1:S2-6.

19. Bae KT, Heiken JP, Brink JA. Aortic and hepatic peak enhancement at CT: effect of contrast medium injection rate--pharmacokinetic analysis and experimental porcine model. Radiology. 1998;206(2):455-64.

20. Bae KT. Peak contrast enhancement in CT and MR angiography: when does it occur and why? Pharmacokinetic study in a porcine model. Radiology. 2003;227(3):809-16.

21. Fleischmann D. Present and future trends in multiple detector-row CT applications: CT angiography. Eur Radiol. Eur Radiol; 2002;12 Suppl 2:S11-5.

22. Brown JR, Robb JF, Block CA, Schoolwerth AC, Kaplan AV, O'Connor GT, et al. Does safe dosing of iodinated contrast prevent contrast-induced acute kidney injury? Circ Cardiovasc Interv. Circ Cardiovasc Interv; 2010;3(4):346-50.

23. Fleischmann D, Hallett RL, Rubin GD. CT angiography of peripheral arterial disease. J Vasc Interv Radiol. 2006;17(1):3-26.

24. Cockcroft DW, Gault MH. Prediction of creatinine clearance from serum creatinine. Nephron. 1976;16(1):31-41.

25. lezzi R, Santoro M, Marano R, Di Stasi C, Dattesi R, Kirchin M, et al. Low-dose multidetector CT angiography in the evaluation of infrarenal aorta and peripheral arterial occlusive disease. Radiology. 2012;263(1):287-98.

26. Fleischmann D, Rubin GD. Quantification of intravenously administered contrast medium transit through the peripheral arteries: implications for CT angiography. Radiology. 2005;236(3):1076-82.

27. Fleischmann D, Chin AS, Molvin L, Wang J, Hallett R. Computed tomography angiography. Radiol Clin North Am. 2015;54(1):1-12.

28. Bae KT, Tran HQ, Heiken JP. Multiphasic injection method for uniform prolonged vascular enhancement at CT angiography: pharmacokinetic analysis and experimental porcine model. Radiology. 2000;216(3):872-80. 


\begin{abstract}
Purpose: To demonstrate the application of a new fast acquisition technique in angiotomography using the maximum capacity of a 160 detector equipment combined with multiphase contrast injection and to compare it with the standard technique for angiotomography of abdominal aorta, iliac arteries and arteries of the lower limbs in relation to diagnostic quality and arterial enhancement. Additionally, we compared the radiation dose and image noise of the two techniques. Methods: We conducted a prospective randomized study with 30 subjects comparing image quality (subjective scale of 1 to 4), arterial enhancement (in Hounsfield Units), image noise (in standard deviation of attenuation) and radiation dose (in mGy.cm) in two groups. A group comprised of the new fast acquisition protocol (Group 1) and the one of widely accepted acquisition, in this study called slow acquisition (Group 2). Results: Groups 1 and 2 were composed of 15 subjects each. We analyzed 187 arterial segments of Group 1, and 190 segments of Group 2. There was no difference between groups in relation to image quality (mean score of $3.60 \pm 0.21$ for fast acquisition and $3.62 \pm 0.21$ for standard acquisition). There was a higher diagnostic quality for the fast acquisition group (99.5\% compared to $96.4 \%)$, although there was less vascular enhancement (426.85UH, compared to 517.67UH). There was a trend towards higher radiation for the fast group (mean of $1296.99+377.44 \mathrm{mGy} . \mathrm{cm}$ compared to $1082.69 \pm 279.74 \mathrm{mGy} . \mathrm{cm}$ ) and lower noise $(20.66 \pm 2.94$ compared to $25.21 \pm 3.21)$. Conclusion: The proposed protocol, optimized for fast angiotomography of the abdominal aorta, iliac arteries and lower limbs arteries has a higher diagnostic quality than the standard protocol. However, we observed lower arterial enhancement not compromising image quality, and a trend towards higher radiation dose (with less noisy images). It should be used on selected patients who may benefit from a faster acquisition.
\end{abstract}

